\title{
Iterative (Turbo) Joint Rate and Data Detection in Coded CDMA Networks
}

\author{
Stefano Buzzi and Stefania Sardellitti \\ Università degli Studi di Cassino \\ 03043 Cassino (FR), Italy \\ buzzi@unicas.it, s.sardellitti@unicas.it
}

Summary. A convolutionally coded CDMA data network wherein each user may transmit at one out of a set of possible data rates is considered in this chapter. The problem of joint detection of the data-rate of each user and of the corresponding information symbols is considered. In particular, it is shown here that the so-called turbo principle can be used also for joint rate and data detection, and indeed an iterative (turbo) procedure is derived wherein the single-user decoders, and the datarate detectors for each user exchange information in order to achieve lower and lower detection error probabilities. Numerical results show that the proposed approach is effective and achieves satisfactory performance.

\section{Introduction}

In this chapter the problem of detecting the data-rate and the information symbols transmitted by each user in a convolutionally coded variable-rate direct-sequence code division multiple access system (DS/CDMA) is considered. Variable-rate CDMA systems wherein each user transmits at one out of a set of available data-rates are nowadays of widespread interest, since they are able to support transmission of heterogeneous kinds of data , such as streaming video, voice, bulk data, etc., with different data-rates. These systems are part of current $3 \mathrm{G}$ wireless networks, and, also, they can support adaptive rate strategies that allow to achieve a larger system throughput $[1,2]$ than a fixed data-rate CDMA systems. Obviously, in a variable-rate CDMA system the receiver generally needs knowledge of the actual data-rate of each user in order to perform data detection, and this information is usually conveyed on a separate multiplexed channel. Alternatively, it may be extracted in some way from the incoming signal, an approach that has been only recently addressed in the literature.

In particular, existing studies in this area have mainly addressed the problem of frame rate detection with regard to a specific second-generation $(2 G)$ or $3 \mathrm{G}$ cellular standard $[3,4]$. A key remark, however, is that none of these 
studies explicitly takes into account the presence of the multiple access interference (MAI). As a further example, the study [5] considers an iterative data detection algorithm for a single user system. More recently, the paper [6] has addressed the problem of blind rate-detection only (i.e. with no joint data detection) for variable data-rate CDMA systems, and the problem of joint detection of the data-rate and of the information symbols in such systems has been considered by the authors in $[7,8]$. In particular, in [7] optimal (i.e. based on the maximum likelihood strategy) non-blind detectors have been proposed; in [8], instead, the focus has been on simpler receiver structures, that are amenable to blind implementations and with a computational complexity that is much lower than that of the receivers in [7].

In this chapter the case in which the users' information symbols are convolutionally coded is considered, and an iterative (turbo) procedure $[9,10]$ is devised in order to obtain reliable estimates of both the data rate and the information symbols for the user of interest. The contribution of this chapter is thus to show that the so-called "turbo principle" can be used also in multiuser joint data-rate and symbol detection with a very attractive performance.

The rest of this chapter is organized as follows. Next section contains the considered system model, while the turbo detection procedure is described in Sect. 3. Finally, numerical results are illustrated in Sect. 4, and conclusions are draws in Sect. 5 .

\section{System Model}

We consider a synchronous convolutionally coded DS/CDMA network with $K$ active users employing BPSK modulation (extension to larger cardinality signaling constellations is trivial); we also assume that the propagation channel introduces frequency-flat fading, that each user may transmit, in each dataframe, at one out of $S$ available data rates $R_{1}<R_{2}<\ldots<R_{S}$, and that the data rates $R_{2}, \ldots, R_{S}$ are all integer multiples of the lowest data-rate $R_{1}$, i.e. we have

$$
R_{i}=m_{i} R_{1}, \quad \forall i=2, \ldots, S
$$

where $m_{i}$ are positive integers. In each frame slot, each user, based on the kind of data to be transmitted, and, possibly, on the propagation channel state, selects one out of the $S$ available data-rates for its transmission. The multirate access scheme that is here considered is the variable spreading length (VSL) [11], i.e. all the signature waveforms have the same chip interval $T_{c}$ and different spreading lengths. In particular, the signatures corresponding to the rate $R_{i}$ have a spreading length equal to $1 /\left(R_{i} T_{c}\right)=T_{b, i} / T_{c}$, with $T_{b, i}$ denoting the symbol interval for the users transmitting at rate $R_{i}$. Based on the above assumptions, the complex envelope of the received signal in a given frame slot is written as 


$$
r(t)=\sum_{k=0}^{K-1} \sum_{p_{k}=0}^{\frac{P r_{k}}{R_{1}}-1} A_{k} \alpha_{k}\left(\left\lfloor p R_{1} / r_{k}\right\rfloor\right) c_{k}\left(p_{k}\right) s_{k}^{r_{k}}\left(t-\frac{p_{k}}{r_{k}}\right)+n(t) .
$$

In this equation, $r_{k}$ is a random variate taking on values in the set $\left\{R_{1}, \ldots, R_{S}\right\}$, and denoting the data-rate of the $k$ th active user, $P$ is the number of data-bits transmitted in each frame by the users at rate $R_{1}$ (thus implying that $P r_{k} / R_{1}$ is the number of bits transmitted by the users at rate $\left.r_{k}\right), c_{k}(\cdot) \in\{+1,-1\}$ denotes the coded bit stream transmitted by the $k$ th user, $\alpha_{k}(\cdot)$ is a complex gain accounting for the channel propagation effects, ${ }^{1}\lfloor\cdot\rfloor$ denotes the usual floor function, while the waveform $n(t)$ represents the ambient noise, that we assume to be a complex zero-mean Gaussian white random process with power spectral density $2 \mathcal{N}_{0}$. Moreover, $s_{k}^{r_{k}}(t)$ and $A_{k}$ are respectively the rate$r_{k}$ signature waveform and the amplitude of the $k$ th user transmitted signal. Denoting by $N_{r_{k}}=1 /\left(r_{k} T_{c}\right)$ the processing gain for the $k$ th user (recall that in the VSL access strategy the processing gain for each user is tied to its data-rate), by $\left\{\beta_{k}^{r_{k}}(n)\right\}_{n=0}^{N_{r_{k}}-1}$ the spreading code (at rate $r_{k}$ ) of the $k$ th user, and by $\phi_{T_{c}}(\cdot)$ a square-root raised cosine bandlimited waveform, we have

$$
s_{k}^{r_{k}}(t)=\sum_{n=0}^{N_{r_{k}}-1} \beta_{k}^{r_{k}}(n) \phi_{T_{c}}\left(t-n T_{c}\right)
$$

assuming that $s_{k}^{r_{k}}(t)$ are unit-energy waveform. At the receiver, the signal $r(t)$ is passed through a filter matched to the waveform $\phi_{T_{c}}(t)$ and sampled at rate $1 / T_{c}$. The resulting samples can be stacked in a $P N_{R_{1}}$-dimensional vector, which we denote by $\boldsymbol{r}$, and which is expressed as

$$
\boldsymbol{r}=\sum_{k=0}^{K-1} \sum_{p_{k}=0}^{P r_{k} / R_{1}-1} A_{k} \alpha_{k}\left(\left\lfloor p R_{1} / r_{k}\right\rfloor\right) c_{k}\left(p_{k}\right) \boldsymbol{s}_{k}^{r_{k}}\left(p_{k}\right)+\boldsymbol{n} .
$$

In the above equation, the vector $s_{k}^{r_{k}}\left(p_{k}\right)$ is the discrete-time version of the waveform $s_{k}^{r_{k}}\left(t-p_{k} / r_{k}\right)$ (notice that this vector, although being $P N_{R_{1}}$ dimensional, has only $N_{r_{k}}$ non-zero entries), while the vector $\boldsymbol{n}$ is the discretetime version of the ambient noise, and is a white complex zero-mean Gaussian random vector with covariance matrix $E\left[\boldsymbol{n} \boldsymbol{n}^{H}\right]=2 \mathcal{N}_{0} \boldsymbol{I}_{P N_{R_{1}}}\left(\right.$ with $(\cdot)^{H}$ denoting conjugate transpose and $\boldsymbol{I}_{n}$ the identity matrix of order $n$ ). Now the $P N_{R_{1}}$-dimensional vector $\boldsymbol{r}$ can be split in $P$ distinct $N_{R_{1}}$-dimensional vectors, $\boldsymbol{r}(0), \ldots, \boldsymbol{r}(P-1)$, i.e. $\boldsymbol{r}=\left[\boldsymbol{r}^{T}(0), \ldots, \boldsymbol{r}^{T}(P-1)\right]^{T}$ with

$$
\boldsymbol{r}(p)=\sum_{k=0}^{K-1} A_{k} \alpha_{k}(p) \boldsymbol{S}_{k}^{r_{k}} \boldsymbol{c}_{k}^{r_{k}}(p)+\boldsymbol{n}(p) \quad p=0, \ldots, P-1
$$

\footnotetext{
${ }^{1}$ Note that we are considering a block fading channel model, i.e. the channel gain is constant over intervals of length not smaller than $T_{b, 1}$.
} 
wherein the $N_{R_{1}} \times\left(r_{k} / R_{1}\right)$-dimensional matrix $\boldsymbol{S}_{k}{ }^{r_{k}}$ is expressed as

$$
\boldsymbol{S}_{k}^{r_{k}}=\boldsymbol{I}_{r_{k} / R_{1}} \otimes\left[\beta_{k}^{r_{k}}(0), \ldots, \beta_{k}^{r_{k}}\left(N_{r_{k}}-1\right)\right]^{T}
$$

with $\otimes$ denoting the Kronecker product and

$$
\boldsymbol{c}_{k}^{r_{k}}(p)=\left[c_{k}\left(p \frac{r_{k}}{R_{1}}\right), \ldots c_{k}\left((p+1) \frac{r_{k}}{R_{1}}-1\right)\right]^{T} .
$$

\section{Detection Algorithm}

A block scheme of the proposed detection algorithm for the $k$ th user is reported in Fig. 1. The vector $\boldsymbol{r}(p)$ is pruned out of the multiple access interference (MAI) ${ }^{2}$ and then is fed to a bank of linear minimum mean square error (MMSE) receivers, each one keyed to one of the available data rates; the output of each MMSE filter is fed to a soft-input-soft-output (SISO) decoder, implementing the well-known BCJR algorithm [12], that gives at the output soft estimates of the coded information symbols. These estimates are then used through the discrete expectation-maximization algorithm [5] in order to take a decision on the most probable data-rate for the $k$ th user. These steps are repeated for each user, so that at the end of each iteration we have the most probable data-rate and the soft estimates of the corresponding coded symbols; these quantities are used at the following iteration in order to build estimates of the multiple access interference, say $\hat{\boldsymbol{m}}_{0}(\cdot), \ldots, \hat{\boldsymbol{m}}_{K-1}(\cdot)$, for each

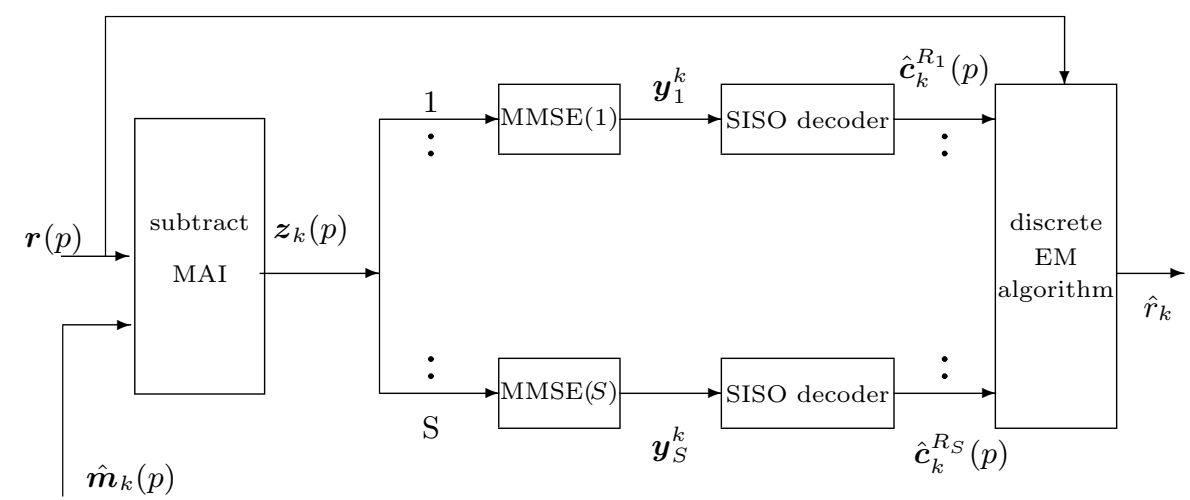

Fig. 1. Block scheme of the $k$ th user receiver

\footnotetext{
${ }^{2}$ More details on how the estimate $\hat{\boldsymbol{m}}_{k}(p)$ of the MAI for the $k$ th user is formed will be given in the sequel of the paper.
} 
user. Note that, obviously, at the beginning of the algorithm no MAI cancellation is carried out (indeed, no MAI estimate is yet available), and the vector $\boldsymbol{r}(p)$ is directly fed to the bank of MMSE filters.

A deeper description of the algorithm is given in the following. We begin with considering the first iteration and, then, the subsequent iterations are described.

\subsection{The Bank of MMSE Filters at the First Iteration}

According to the detection strategy proposed in [8], the data vector $\boldsymbol{r}(p)$ is fed to a bank of MMSE filters, each keyed to one of the available data rates. Denoting by $\boldsymbol{D}_{n}^{k}(p)$ the MMSE filter for the $k$ th user and keyed to the $n$th data rate (with $n=1, \ldots, S$ ), and denoting by $\boldsymbol{y}_{n}^{k}(p)$ the output of this MMSE filter, ${ }^{3}$ we have

$$
\boldsymbol{y}_{n}^{k}(p)=\boldsymbol{D}_{n}^{k H}(p) \boldsymbol{r}(p), \quad n=1, \ldots, S
$$

with $(\cdot)^{H}$ denoting conjugate transpose and

$$
\boldsymbol{D}_{n}^{k}(p)=A_{k} \alpha_{k}(p) \boldsymbol{R}_{k, n}^{-1} \boldsymbol{S}_{k}^{R_{n}} .
$$

In the above equation, $\boldsymbol{R}_{k, n}$ is the data covariance matrix under the hypothesis that the $k$ th user is transmitting at rate $R_{n}$, i.e. we have

$$
\boldsymbol{R}_{k, n}=A_{k}^{2} \sigma_{\alpha_{k}}^{2} \boldsymbol{S}_{k}^{R_{n}} \boldsymbol{S}_{k}^{R_{n} H}+\boldsymbol{M}_{k}
$$

with

$$
\boldsymbol{M}_{k}=\frac{1}{S} \sum_{j \neq k} \sum_{\ell=1}^{S} A_{j}^{2} \sigma_{\alpha_{j}}^{2} \boldsymbol{S}_{j}^{R_{\ell}} \boldsymbol{S}_{j}^{R_{\ell} H}+2 \mathcal{N}_{0} \boldsymbol{I}_{N_{R_{1}}} .
$$

the covariance matrix of the $k$ th user's MAI plus thermal noise. In the above equation, $\sigma_{\alpha_{j}}^{2}$ is the mean square value of the channel coefficient $\alpha_{j}(p)$. Note that in (10) the covariance matrix of the MAI has been averaged with respect to the data rates of the interfering users too; indeed, at the first iteration no prior information on the data rates of the interfering users is available, and thus averaging with respect to the available data rates seems to be the only reasonable option.

\subsection{The SISO Decoders at the First Iteration}

The output of each MMSE filter is then fed to a bank of SISO decoders implementing the BCJR algorithm [12]. For the sake of brevity, we do not dwell on the description of this algorithm, that can be found in many digital

\footnotetext{
${ }^{3}$ Note that for $n=1 \boldsymbol{y}_{n}^{k}(p)$ is a scalar quantity and not a vector; likewise, $\boldsymbol{D}_{n}^{k}(p)$ for $n=1$ is a vector and not a matrix.
} 
communications textbooks. What suffices to say here is that at the output of each SISO decoder we have soft estimates for the coded information symbols for each rate hypothesis. Otherwise stated, at the output of the SISO decoder on the $k$ th user's receiver' $n$th finger, we have the soft estimates $\hat{c}_{k}^{R_{n}}(0), \hat{c}_{k}^{R_{n}}(1), \ldots$ of the information symbols for the hypothesis that the user $k$ is transmitting at the rate $R_{n}$.

\subsection{The Rate Estimation at the First Iteration}

The outputs of the SISO decoders are then fed to a discrete EM algorithm that takes a decision on the most likely data-rate for the $k$ th user according to the maximum likelihood rule

$$
\hat{r}_{k}=\arg \max _{r_{k}} E_{c_{k}^{r_{k}}(\cdot)}\left[\log p\left(\boldsymbol{r}(p) \mid \boldsymbol{c}_{k}^{r_{k}}(\cdot), r_{k}\right)\right]
$$

with $E_{c_{k}^{r_{k}(\cdot)}}[\cdot]$ denoting statistical expectation with respect to the coded information symbols, and $p(\boldsymbol{r}(p) \mid \cdot)$ denoting the conditional likelihood of the data vector. Since the data vector $\boldsymbol{r}(p)$ is conditionally Gaussian, upon letting $\boldsymbol{Q}_{k, n}=\boldsymbol{S}_{k}^{R_{n}{ }^{H}} \boldsymbol{M}_{k}^{-1} \boldsymbol{S}_{k}^{R_{n}}$, it can be shown that maximization (11) is equivalent to

$$
\begin{gathered}
\hat{r}_{k}=\arg \max _{r_{k}}\left[-\boldsymbol{r}^{H}(p) \boldsymbol{M}_{k}^{-1}(p) \boldsymbol{r}(p)\right. \\
+2 \Re\left(\boldsymbol{r}^{H}(p) \boldsymbol{M}_{k}^{-1}(p) A_{k} \alpha_{k}(p) \boldsymbol{S}_{k}^{R_{n}} \hat{\boldsymbol{c}}_{k}^{R_{n}}(p)\right)-A_{k}^{2}\left|\alpha_{k}(p)\right|^{2} \\
\left.\times\left(\sum_{i, j=1, i \neq j}^{m_{n}} \hat{c}_{k}^{R_{n}}(i) \hat{c}_{k}^{R_{n} *}(j) \boldsymbol{Q}_{k, n}(i, j)+\operatorname{trace}\left(\boldsymbol{Q}_{k, n}\right)\right)\right] .
\end{gathered}
$$

\subsection{The Following Iterations}

At the end of the first iteration we have, for each user, a partial estimate $\hat{r}_{k}$ of its rate and a soft estimate of the corresponding coded symbols, i.e. $\hat{c}_{k}^{\hat{r}_{k}}(0), \hat{c}_{k}^{\hat{r}_{k}}(1), \ldots$. At the $k$ th user receiver, MAI is cancelled according to the relation

$$
\boldsymbol{z}_{k}(p)=\boldsymbol{r}(p)-\sum_{j \neq k} A_{j} \boldsymbol{S}_{j}^{\hat{r}_{j}} \boldsymbol{c}_{j}^{\hat{r}_{j}}(p) \alpha_{j}(p)
$$

i.e. we have

$$
\begin{gathered}
\boldsymbol{z}_{k}(p)=A_{k} \alpha_{k}(p) \boldsymbol{S}_{k}^{r_{k}} \boldsymbol{c}_{k}^{r_{k}} \\
+\sum_{j \neq k} A_{j} \alpha_{j}(p)\left(\boldsymbol{S}_{j}^{r_{j}} \boldsymbol{c}_{j}^{r_{j}}-\boldsymbol{S}_{j}^{\hat{r}_{j}} \boldsymbol{c}_{j}^{\hat{r}_{j}}\right)+\boldsymbol{n}(p) .
\end{gathered}
$$

The output of the MMSE filter is now written as

$$
\boldsymbol{y}_{n}^{k}(p)=\boldsymbol{D}_{n}^{k H}(p) \boldsymbol{z}_{k}(p), \quad n=1, \ldots, S
$$


with

$$
\boldsymbol{D}_{n}^{k}(p)=A_{k} \alpha_{k}(p) \boldsymbol{G}_{k, n}^{-1} \boldsymbol{S}_{k}^{R_{n}} .
$$

In the above equation, $\boldsymbol{G}_{k, n}$ is the covariance matrix of $\boldsymbol{z}_{k}(p)$ under the hypothesis that the $k$ th user is transmitting at rate $R_{n}$, and given the available estimates of the data rates and coded symbols for the interfering users, i.e.

$$
\begin{aligned}
& \boldsymbol{G}_{k, n}=A_{k}^{2} \sigma_{\alpha_{k}}^{2} \boldsymbol{S}_{k}^{R_{n}} E\left[\boldsymbol{c}_{k}^{R_{n}}(p) \boldsymbol{c}_{k}^{R_{n} H}(p)\right] \boldsymbol{S}_{k}^{R_{n} H}+2 \mathcal{N}_{0} \boldsymbol{I}_{N_{R_{1}}} \\
& +\sum_{j \neq k} A_{j}^{2} \sigma_{\alpha_{j}}^{2} \boldsymbol{S}_{j}^{\hat{r}_{j}}\left(\boldsymbol{I}_{\hat{r}_{j} / R_{1}}-\operatorname{diag}\left(\boldsymbol{c}_{j}^{\hat{r}_{j}}(p) \odot \boldsymbol{c}_{j}^{\hat{r}_{j} *}(p)\right) \boldsymbol{S}_{j}^{\hat{r}_{j} H} .\right.
\end{aligned}
$$

In the above equation, $\odot$ denotes componentwise product, while the statistical expectation $E\left[\boldsymbol{c}_{k}^{R_{n}}(p) \boldsymbol{c}_{k}^{R_{n} H}(p)\right]$ is such that its $(i, j)$ th entry is equal to 1 for $i=j$ and is equal to $\hat{\boldsymbol{c}}_{k}^{R_{n}}(i) \hat{\boldsymbol{c}}_{k}^{R_{n} *}(j)$ for $i \neq j$.

\section{Numerical Results}

In this section we show some numerical results on the performance of the proposed detection algorithm. In Figs. 2 and 3 the probability of erroneous rate detection (PERD) and of bit error probability conditioned on correct rate

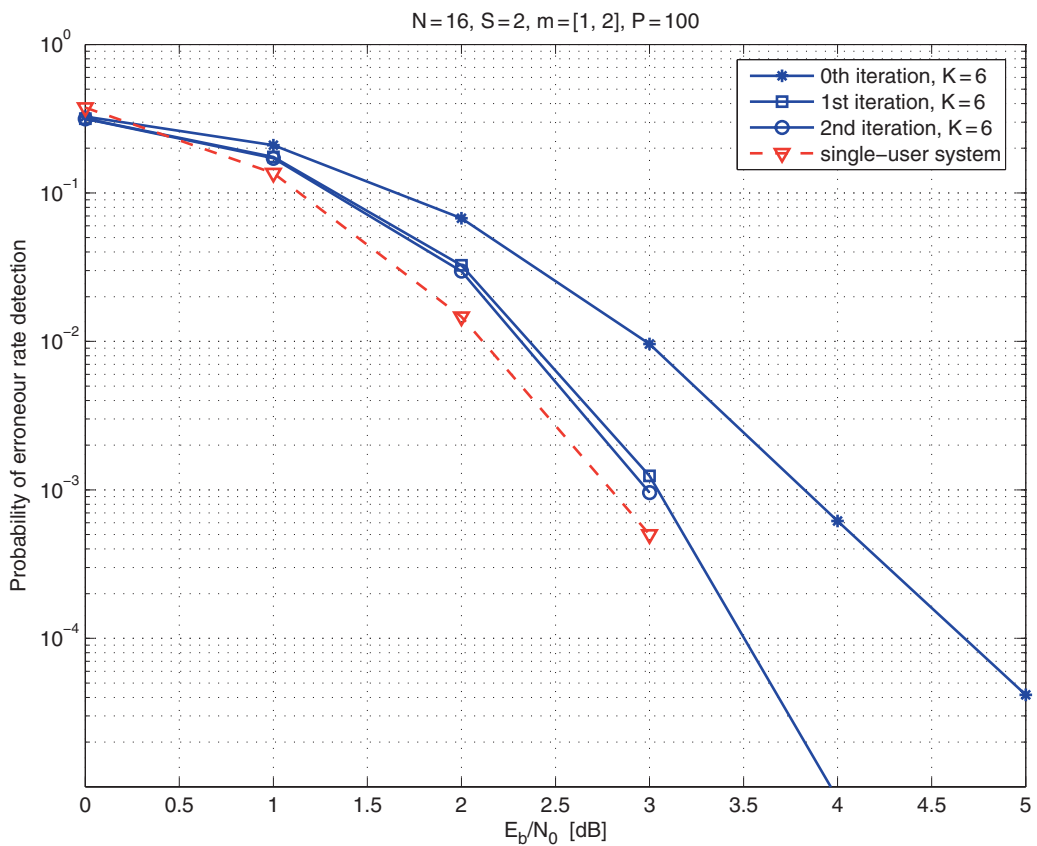

Fig. 2. Probability of erroneous rate detection vs. the received energy contrast 


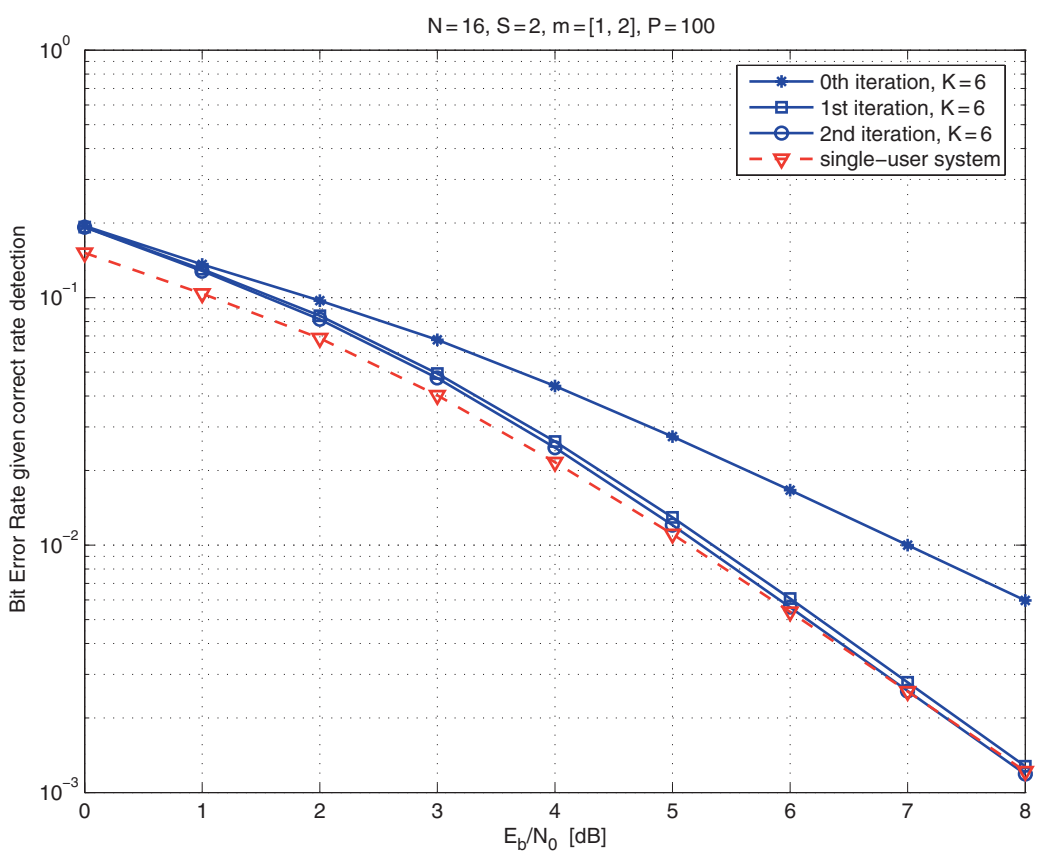

Fig. 3. Probability of bit error given correct rate detection vs. the received energy contrast

detection (BER $\mid \mathrm{CRD})$ vs. the received energy contrast $E_{b} / \mathcal{N}_{0}$ has been reported. In particular a DS/CDMA system with data frames of length $P=100$ on a frequency-flat Rayleigh fading channel has been considered. The largest processing gain is $N_{R_{1}}=16$, and $S=2$ data rates with a rate ratio vector given by $\boldsymbol{m}=[1,2]$ has been used. The number of users is $K=6$, while a convolutional code of rate $1 / 2$ has been used. For comparison purposes we also report the curve corresponding to the performance of a single-user system. It can be noted that the turbo rate detection strategy achieves in only one iteration a performance very close to that of a single user system, i.e. the proposed detection strategy achieves almost ideal interference cancellation. From Fig. 3, it is also seen that the proposed system bit error rate also attains the single user bound.

Overall it can be stated that the proposed procedure is very effective and is able to get rid of the multiple access interference achieving performance levels close to that of a single user system.

\section{Conclusions}

In this chapter an iterative multiuser joint data-rate and information symbol detection algorithm for coded DS/CDMA networks has been proposed. 
The proposed receiver structure consists, for each user, of a bank of MMSE receivers, each one matched to one of the available data-rates, followed by a bank of SISO decoders, and, finally, by a discrete EM algorithm that chooses, for each user, the most probable data-rate at that iteration. The estimated data rates and coded information symbols provided at the end of each iteration are used in the following one in order to cancel multiple access interference and to improve the detection reliability. Numerical results have shown that the proposed strategy is very effective, thus proving that the turbo principle can be successfully exploited in order to detect the data-rate and the information symbols in a coded CDMA network.

\section{References}

1. A. Goldsmith and S. Chua, "Adaptive coded modulation for fading channels," IEEE Trans. Commun., Vol. 46, pp. 595-602, May 1998

2. S. A. Jafar and A. Goldsmith, "Adaptive multirate CDMA for uplink throughput maximization," IEEE Trans. Wireless Commun., Vol. 2, pp. 218-228, March 2003

3. G. Yang and S. Kellel, "Optimal frame rate detection for CDMA mobile stations with variable-rate data transmission," Proc. of 1999 Fifth Asia-Pacific Conf. Commun. and Fourth Optoelectron. and Commun. Conf., Bejing, China, October 1999

4. I. Held and A. Chen, "Reduced complexity blind rate detection for second and third generation cellular CDMA systems," Proc. 1999 IEEE Global Commun. Conf. (GLOBECOM '99), Rio de Janeiro (Brasil), December 1999

5. H. Wymeersch and M. Moeneclaey, "ML-based blind symbol rate detection for multi-rate receivers," Proc. of 2005 IEEE Int. Conf. Commun. (ICC 2005), Vol. 3, pp. 2092-2096, May 2005

6. S. Buzzi and A. De Maio, "Code-aided blind detection of the transmission rate for multirate direct-sequence CDMA systems," IEEE Trans. Signal Process., Vol. 53, pp. 2182-2192, June 2005

7. S. Buzzi and S. Sardellitti, "Joint rate and data detection in variable-rate CDMA systems," Proc. of IEEE Radio and Wireless Symp. (RWS '06), San Diego (CA), USA, January 2006

8. S. Buzzi and S. Sardellitti, "Blind MMSE-based receivers for rate and data detection in variable-rate CDMA systems," Proc. of the 14th European Signal Process. Conf., Florence, Italy, September 2006

9. X. Wang and H. V. Poor, "Iterative (Turbo) soft interference cancellation and decoding for coded CDMA," IEEE Trans. Commun., Vol. 47, pp. 1046-1061, July 1999

10. N. Noels et al., "Turbo synchronization: an EM algorithm interpretation," Proc. of 2003 IEEE Int. Conf. Commun. (ICC 2003), Vol. 4, pp. 2933-2937, May 2003

11. S. Buzzi, M. Lops and A.M. Tulino, "Blind adaptive multiuser detection for asynchronous dual-rate DS/CDMA systems," IEEE J. Selected Areas Commun., Vol. 19, pp. 233-244, February 2001

12. L. R. Bahl, J. Cocke, F. Jelinek and J. Raviv, "Optimal decoding of linear codes for minimizing symbol error rate," IEEE Trans. Inform. Theory, Vol. 20, pp. 284-287, March 1974 